Archives de sciences sociales des religions

131-132 | juillet - décembre 2005

Varia

\title{
Des funérailles de l'entre-deux Rituels funéraires des migrants Manjak en France
}

\section{Agathe Petit}

\section{(2) OpenEdition}

1 Journals

Édition électronique

URL : http://journals.openedition.org/assr/3256

DOI : 10.4000/assr.3256

ISSN : $1777-5825$

Éditeur

Éditions de l'EHESS

\section{Édition imprimée}

Date de publication : 1 décembre 2005

Pagination : 87-99

ISBN : 2-7132-2045-9

ISSN : 0335-5985

\section{Référence électronique}

Agathe Petit, « Des funérailles de l'entre-deux Rituels funéraires des migrants Manjak en France », Archives de sciences sociales des religions [En ligne], 131-132 I juillet - décembre 2005, mis en ligne le 30 juin 2008, consulté le 30 avril 2019. URL : http://journals.openedition.org/assr/3256 ; DOI 10.4000/assr.3256

Ce document a été généré automatiquement le 30 avril 2019

() Archives de sciences sociales des religions 


\title{
Des funérailles de l'entre-deux Rituels funéraires des migrants Manjak en France
}

\author{
Agathe Petit
}

À propos des Manjak

1 Le groupe manjak reste à de nombreux points de vue mal connu et les données bibliographiques en langue française sont rares ${ }^{1}$. Les migrants manjak sont originaires $\mathrm{du}$ nord de la Guinée-Bissau. Ils sont souvent assimilés à tord aux migrants sénégalais car bon nombre de candidats au départ ont d'abord migré vers le Sénégal afin de pouvoir profiter des accords de main-d'œuvre avec la France et ont de ce fait adopté la nationalité sénégalaise. Les Manjak comptent parmi les pionniers de l'immigration africaine et constituent un groupe numériquement important en France, surtout en région parisienne et dans le sud-est de la France (en particulier les agglomérations marseillaise et toulonaise). Par rapport à d'autres groupes de migrants sénégambiens (Soninké, Haalpular, Wolof, etc.), ils ont la particularité d'avoir très tôt privilégié le regroupement familial et certaines familles sont implantées en France depuis trois, voire quatre, générations ; en dépit d'une migration désormais ancienne, ils restent très attachés à leur religion « coutumière » et au culte des ancêtres qui se mêlent, pour bon nombre d'entre eux, à une pratique soutenue de la religion catholique et un investissement au sein des « communautés chrétiennes africaines ${ }^{2}$ mises en place dans certaines paroisses.

2 Les Manjak, migrants ouest-africains des premières heures, représentent une population immigrée et issue de l'immigration installée durablement en France, qui non seulement y travaille et y vit, mais y meurt aussi. Il convient donc de s'intéresser à une question souvent négligée dans les travaux en sciences sociales sur les migrations, celle du devenir post-mortem des immigrés et de la manière dont ils gèrent un décès survenu en migration, d'autant que les Manjak ont une conception et un traitement de la mort profondément différents des nôtres.

3 Les ancêtres occupent une place prépondérante et sont les garants de l'intégrité familiale et de la coutume. Ce sont des êtres auxquels on se réfère et qu'on honore au moyen de 
rites appropriés, autant pour les tenir à l'écart du monde des vivants que pour solliciter leur intervention. Dans les villages d'origine, chaque concession familiale compte en son sein un espace dédié aux ancêtres où chacun est représenté par un pieu de bois, appelé picap, plus ou moins anthropomorphique. Cet autel des ancêtres est le lieu de référence du groupe familial mais aussi un espace cérémoniel central et le pivot de la vie sociale. Il existe une relation étroite entre les ancêtres et leur descendance attestée à travers des rites réguliers et des gestes quotidiens. Avant tout rituel impliquant un des membres de la maisonnée, des libations doivent être effectuées sur les picap pour inciter les Balugum (ancêtres) à veiller au bon déroulement de la cérémonie ; on leur offre des cadeaux, ils sont associés aux fêtes par le versement de l'alcool partagé mais également aux repas. En dehors des fréquentes prières qu'on leur adresse, un vrai dialogue est maintenu avec eux : ils sont consultés, par l'intermédiaire d'un médium ou d'un devin, en cas de problèmes et sont informés des événements familiaux.

Les rituels funéraires manjak s'articulent autour de plusieurs grandes séries de rites. À la mort d'un individu débute la première période appelée Ka lotan pöm (traversée du mort) destinée à la préparation du corps et à l'interrogation du défunt (pour les Manjak, toute mort, à l'exception de celle du vieillard, est suspecte: on ne meurt jamais de mort naturelle), puis à son inhumation. Après une période variable, qui dépend des moyens dont dispose la famille, ont lieu les "grandes funérailles » (P-moy), deuxième phase du dispositif rituel. Cette cérémonie festive est destinée à honorer le défunt, à marquer son changement de statut et ainsi à mettre en place de nouveaux types de liens entre les vivants et le défunt. Les grandes funérailles sont l'une des étapes essentielles du processus d'ancestralisation, même si celui-ci n'est mené à terme qu'à la suite de l'intégration du picap (ou pe-ciap, pëcap), image ancestralisée du défunt, au sein de l'autel des ancêtres. Ériger le picap est l'acte fondateur du culte des ancêtres. Ces rituels revêtent un double caractère: ils servent dans un premier temps à honorer le défunt et à déconstruire son image (du statut de vivant, il passe à celui de défunt), mais surtout, sont destinés à lui ouvrir les portes du monde des ancêtres et à construire sa nouvelle identité dans l'au-delà (de défunt, il devient ancêtre). Si ceux-ci n'ont pas été menés à terme, l'âme du défunt erre dans le village, y revient pour des visites, signe qu'il lui manque quelque chose, ou s'en prend à ses descendants.

Que deviennent ces dispositifs rituels dans un contexte de migration? L'aménagement des pratiques cérémonielles en situation d'immigration, et plus encore des pratiques funéraires, est un champ de recherche encore peu exploré. Or, la variabilité des formes de la fête, et par extension des dispositifs rituels, largement mise en avant dans des travaux récents (Hérault, 1992; Segalen, 1998), s'applique tout particulièrement au contexte migratoire. Les pratiques funéraires qui ont cours dans la culture d'origine, elles-mêmes susceptibles de se modifier, ne peuvent être transposées à l'identique du fait des nombreuses contraintes liées au nouveau cadre de vie qui combine urbanisation et migration (Brisebarre, 1998; Dessing, 2001). Ces pratiques, qui confrontent ou font coexister deux systèmes de références culturelles différents, se jouent en plusieurs temps, sur plusieurs espaces : ici et là-bas, entre ici et là-bas. Il s'agit d'une situation originale qui bouleverse les normes et compromet leur unité : les unités de temps, de lieu et d'action sont éclatées. Cette disjonction entraine une déstructuration et une discontinuité dans le déroulement séquentiel des funérailles et oblige à des négociations et à des remaniements. Comment se structurent les funérailles en migration? À quelles contraintes doivent faire face les migrants qui gèrent un décès en France? Quelles 
stratégies de résistance mettent-ils en œuvre? Assiste-t-on à des aménagements, des glissements de sens et à une réinterprétation des rôles? Les différentes formes d'adaptations, de (re)négociations dans l'agencement séquentiel confèrent-elles aux rituels une symbolique supplémentaire?

Récit : les funérailles d'Antoine Mendy entre Marseille, Dakar et Kanow, village manjak

Antoine Mendy, ancien navigateur, était installé avec sa famille à Marseille. Il est décédé à 90 ans à l'hôpital. Avec l'accord de son médecin traitant, son corps a pu être ramené au domicile de sa fille où il vivait.

7 C'est dans la «maison mortuaire » que se sont tenues les veillées funèbres et réunions: les visiteurs y sont venus rendre un dernier hommage au défunt. Un des aînés de la " communauté » manjak, à qui l'on confie généralement la toilette des hommes parce qu'il connaît les gestes à accomplir, les paroles à prononcer, est venu laver le corps et l'habiller. Jusqu'à l'enterrement, le corps est resté dans la chambre funéraire. La nouvelle du décès a circulé au sein du groupe et jusqu'à l'inhumation, la maison n'a pas désempli. Le soir du décès et la veille de l'enterrement ont eu lieu deux grandes veillées traditionnelles. Les femmes ont revêtu leur tenue de cérémonie, ont dansé, chanté pour célébrer la mort de ce vieil homme, considéré comme l'un des plus éminents griots manjak de la région...

Chaque visiteur est tenu, en plus de sa contribution financière qui accompagne la présentation des condoléances, d'apporter une chemise et/ou une bouteille d'alcool. La première s'ajoute aux effets personnels du défunt pour constituer la " valise mortuaire » tandis que la seconde est destinée aux libations. Effectivement, les visiteurs viennent rendre un dernier hommage au défunt et s'adressent à lui par l'intermédiaire d'un officiant, généralement un proche du défunt, qui, par des libations, demande au défunt d'offrir sa protection à ses proches parents et à l'intéressé mais aussi de rester en paix en attendant de rejoindre le monde des ancêtres.

9 Jusqu'à ce que le défunt soit intégré à l'autel des ancêtres dans la concession familiale au pays, il est considéré comme le seul à pouvoir protéger son entourage des influences néfastes et des attaques sorcellaires. À côté de ces veillées traditionnelles qui sont réapparues il y a peu au sein du groupe, sont organisées des veillées de prières, sous la direction d'un homme pieux, au cours desquelles on récite des chapelets, des prières, on chante, on lit des passages de la Bible.

10 L'enterrement a eu lieu le samedi suivant le décès, soit près d'une semaine plus tard. Il a occasionné un rassemblement important : plusieurs centaines de personnes sont venues de la région, des autres villes de migration de France, du Portugal ou d'Allemagne. Il n'est pas rare de voir certains membres de la famille qui résident au Sénégal faire le déplacement jusqu'en France pour venir assister à un enterrement, si toutefois ils en ont les moyens financiers. Il est vivement recommandé à tous les proches de venir assister aux funérailles. C'est d'ailleurs pour cette raison qu'il est d'usage d'attendre plusieurs jours avant de procéder à l'inhumation afin que tous les proches soient informés du décès et puissent faire le déplacement, sous peine de s'exposer à des sanctions. La célébration a eu lieu dans la paroisse du défunt et a laissé une large place à l'intervention de laïcs membres de la «communauté chrétienne africaine » qui ont bien connu le défunt. La chorale de la communauté est également venue pour chanter des cantiques. Après l'inhumation dans la "Cathédrale du Silence " ${ }^{3}$, conformément au souhait du défunt, l'assistance a été invitée à se réunir dans une salle louée pour l'occasion. Des moutons et des cochons ont été achetés pour l'occasion afin d'effectuer des sacrifices et de nourrir les 
convives. Les libations se sont poursuivies et de grandes quantités d'alcool ont été consommées en ces occasions. Ceux qui ne sont pas venus à la maison mortuaire profitent de ce rassemblement pour venir présenter leurs condoléances à la famille suivant les mêmes modalités. Huit jours plus tard, une messe a été célébrée à la mémoire du défunt ${ }^{4}$. Une autre messe d'anniversaire, un an après le décès, marque la fin de la période de deuil.

Peu de temps après la messe anniversaire, le fils aîné du défunt est parti dans le village d'origine de son père, Kanow, accompagné d'un de ses cousins dakarois, ramener la valise mortuaire, forme de retour symbolique du défunt dans son village natal. Une grande cérémonie festive a été organisée à cette occasion. Trois ans plus tard, la fille aînée du défunt, après de nombreuses pérégrinations administratives en vue de l'obtention de ses papiers d'identité, est partie à son tour à Kanow afin d'ériger le picap de son père. Les enfants n'étaient jamais repartis ni au Sénégal, ni en Guinée-Bissau. depuis leur arrivée en France dans les années cinquante. L'intégration de l'effigie du défunt au sein de l'autel familial marque son entrée dans le monde des ancêtres mais également la fin du cycle funéraire. Toutefois, il est recommandé de se rendre sur l'autel un an après l'intégration du nouvel ancêtre afin de réaliser un sacrifice et par la suite de célébrer les ancêtres lors de la fête annuelle des morts. Cependant, ni l'un ni l'autre des enfants, aujourd'hui âgés, ne sont encore retournés au village pour se rendre sur l'autel de leurs ancêtres. Quoi qu'il en soit, le défunt a rejoint l'autre monde et son esprit peut voyager librement entre le village, la France... Ses proches continuent de l'honorer quotidiennement : avant chaque repas, avant de boire, ils « versent » par terre pour lui rendre hommage et s'assurer de sa protection.

Enterrement ici, funérailles là-bas

Lorsqu'un décès survient en France, les immigrés, quelle que soit leur origine, ont le choix entre plusieurs modalités de sépulture : l'inhumation sur place, si possible dans un carré musulman si telle était la confession du défunt, l'inhumation dans le pays d'origine après un rapatriement de la dépouille mortelle, ou encore la crémation. Bon nombre de musulmans sénégalais préfèrent rapatrier les dépouilles mortelles dans le pays d'origine afin que le défunt soit inhumé au village. Les Manjak, eux, sont le plus souvent hostiles au transfert posthume du corps qu'ils jugent trop coûteux et peu conforme à leur situation migratoire ${ }^{5}$. Les migrants manjak, dont le projet migratoire a privilégié une installation durable dans le pays d'immigration et un regroupement familial précoce, optent généralement pour une inhumation en France, à la différence d'autres groupes de migrants.

Alors que les rites manjak qui accompagnent l'inhumation ne constituent pas une phase importante lorsqu'ils ont lieu au village, en migration ce premier temps funéraire prend une valeur et une ampleur inédites car c'est la seule séquence qui est réalisée en France. L'enterrement et les cérémonies qui l'accompagnent occasionnent des rassemblements importants et l'espace de sépulture prend en migration une importance nouvelle puisque, dans les villages d'origine les tombes ne font l'objet ni d'attentions particulières, ni d'un quelconque culte du souvenir. Le cycle funéraire se poursuit suivant un mouvement de va-et-vient entre la France et l'Afrique. Les "grandes» funérailles et les rites d'ancestralisation sont célébrés au village. Le rapatriement de la valise mortuaire (u nema ), constituée des objets et effets personnels du défunt, fait le lien entre les deux espaces funéraires. Il ne s'agit pas d'une simple valise qui servirait à ramener les effets personnels du défunt sur sa terre d'origine. Tout est fait pour donner à cette valise des airs de cercueil: une croix et le certificat de décès sont apposés sur celle-ci. Si elle vient 
désormais confirmer le décès et non plus l'annoncer comme autrefois, elle le matérialise, compense l'inhumation en France et surtout consacre le retour de l'âme du défunt sur la terre de ses ancêtres. Elle sert de support aux cérémonies funéraires qui ont lieu au village. Le rapatriement de la valise peut avoir lieu à partir du huitième jour qui suit le décès mais dépend finalement des moyens financiers dont dispose la famille. Le plus souvent, il faut plusieurs années pour réaliser ce retour qui exige de nombreuses dépenses. Il est assuré par un proche parent, désigné par l'individu de son vivant ou par l'entourage à l'issue d'un rituel de divination.

Ce voyage, qui célèbre en quelque sorte le retour du défunt dans le pays de ses ancêtres à travers une forme de retour symbolique et lui ouvrira les portes de l'au-delà, se déroule le plus souvent en plusieurs étapes : en premier lieu, en France où la famille rassemble ses proches et amis pour célébrer le départ de la valise, puis à Dakar où sont célébrées des messes et où les proches parents qui y vivent réalisent des sacrifices et, à travers des rites de divination, s'assurent que la route vers le village est « ouverte », l'accompagnateur est alors lui-même accompagné par un proche parent dakarois qui connaît bien les coutumes $\mathrm{du}$ village et sert de guide; si une partie de la famille proche vit en Casamance, ces accompagnateurs s'y arrêtent et y effectuent également des sacrifices, et enfin au village d'origine en Guinée-Bissau. Ces étapes ne sont pas anodines et entraînent des dépenses considérables, supportées par les émigrés et la famille du défunt.

Les cérémonies qui accompagnent le retour de la valise remplacent, au village, les rituels d'inhumation et par conséquent la première phase des funérailles. Elles constituent une séquence supplémentaire propre à la migration qui vient compenser une inhumation réalisée en France. Le rapatriement de la valise au village marque le début des rituels qui accompagnent le défunt dans son changement de statut et dans l'accès à l'au-delà. La cérémonie finale qui consiste en l'intégration de l'esprit du défunt au monde des ancêtres, par la construction du picap, pourra être ajournée si la famille ne dispose pas des moyens nécessaires à son organisation, ce qui est le plus souvent le cas. La valise est alors considérée comme l'incarnation temporaire de l'esprit du défunt et pallie l'absence d'effigie du défunt au sein de l'autel des ancêtres. Elle est conservée précieusement dans la maison familiale tandis que les parents du défunt venus de France repartent en attendant que puisse se poursuivre le cycle funéraire. La troisième et dernière étape du cycle funéraire, marque l'ancestralisation du défunt et a lieu dans le village d'origine mais en présence d'au moins un proche parent émigré.

16 Tandis qu'au village, seule l'âme du défunt bénéficie d'un traitement rituel de la part des vivants, en migration, le corps du défunt est le support de nouvelles formes d'expression du souvenir des morts et un attachement inédit aux sépultures prend forme.

De la privation des funérailles à la reconstruction d'une convivialité cérémonielle

D'un point de vue social, les cérémonies familiales ont, en Afrique, un caractère public très marqué. Rien de privé ne peut advenir sans un investissement public à la fois économique et relationnel. Au niveau spatial, les conditions de vie en milieu urbain viennent accentuer ce caractère public. En effet, tandis que dans les villages d'origine, le cadre des rituels funéraires est en général celui de la concession familiale qui permet des rassemblements importants, en milieu urbain, les limitations des espaces domestiques occasionnent souvent des débordements sur l'espace public. À l'inverse, en France, les pratiques funéraires en milieu urbain se caractérisent par la privatisation, la désocialisation et l'anonymat. Les dispositifs rituels des migrants n'échappent pas à cette 
tendance étroitement liée aux conditions de logement et aux relations sociales dans les grandes agglomérations.

Durant les premières décennies de leur migration, les pratiques des Manjak sont effectivement allées dans le sens d'une privatisation et souvent d'une modération de la participation, et dans tous les cas d'une limitation de leur durée et de leur intensité. Aujourd'hui, les cérémonies d'enterrement d'un migrant manjak occasionnent des rassemblements importants. Assister aux funérailles est devenu une obligation à laquelle il est difficile de se soustraire. Les cérémonies familiales, et en premier lieu les funérailles, sont devenues une des occasions de se retrouver et ont contribué à la réaffirmation et à la consolidation des liens sociaux. L'ampleur de ces rassemblements est liée d'une part à la présence d'une majorité de familles qui ont su, au fil de leur insertion sur le territoire d'immigration et à la faveur de certains changements concernant leur cadre de vie, reconstruire des réseaux de relations, amicaux et un système d'obligations et d'échanges cérémoniels qui, même s'ils sont moins visibles, occupent tout de même une place importante, et d'autre part aux contraintes sociales liées à la participation aux funérailles. Les funérailles manjak égalent désormais celles auxquelles on peut assister dans le pays d'origine à l'occasion des " grandes funérailles ».

Alors qu'en France les personnels hospitaliers et des sociétés de pompes funèbres sont devenus des acteurs à part entière et interviennent à tous les niveaux dans la préparation du défunt et l'organisation des funérailles, les familles manjak endeuillées insistent pour que le corps du défunt soit ramené au domicile lorsque le décès survient à l'hôpital afin que les veillées mortuaires puissent avoir lieu en présence du corps du défunt et que la toilette puisse être réalisée suivant les usages coutumiers. Malgré un impossible confinement strictement villageois ou familial dans la gestion d'un décès survenu en France, la famille ou l'entourage se réapproprie certaines séquences et notamment celles liées à la préparation du cadavre. Cette prise en charge interne témoigne de la volonté de faire face à la professionnalisation des pratiques funéraires en France et de préserver certaines pratiques et savoir-faire coutumiers mais aussi de renforcer la cohésion du groupe autour de la famille endeuillée. Mais cette volonté pose le problème de la compétence des acteurs et de la reproduction de certains rôles et fonctions en migration. Dans les régions d'origine, les dispositifs rituels sont ponctués de pratiques, gestes, paroles, chants qui relèvent de la coutume. Les rôles des protagonistes sont répartis en fonction de relations d'obligations statutaires entre lignages et d'un système d'obligations réciproques au sein de la famille.

$\mathrm{Au}$ village, l'organisation des cérémonies est généralement confiée aux aînés. Or, les migrants sont le plus souvent de jeunes adultes : ils ont pour la plupart quitté le village avant que ne leur soient confiées des responsabilités dans le cadre de l'organisation des cérémonies ou, pour certains, avant même qu'ils aient assisté et participé activement à celles-ci. Ils ne sont pas, comme les anciens, imprégnés par des dispositifs rituels qu'ils auraient vus maintes fois se dérouler. Ils ne taisent d'ailleurs pas leur ignorance quant au déroulement séquentiel de ces dispositifs et à toutes les pratiques qui les accompagnent. La migration précoce entraîne un manque de références, de modèles sur lesquels pourrait s'appuyer la mise en œuvre des rituels.

21 Au cours des cérémonies sociales - veillées, condoléances, libations... - il existe également un ensemble de rôles déterminés en fonction de l'appartenance sociale et statutaire. Au village, la préparation et l'embaumement du défunt revient à l'« habilleur des morts » ( natema pom) qui réalise les gestes et sacrifices appropriés, l'inhumation et les rites qui 
l'accompagnent sont confiés aux «fossoyeurs » (ba-guran) tandis que l'interrogatoire du mort (u-cos pom) revient aux hommes et femmes de la maison (ban-toy) détenteurs des pouvoirs de divination. L'interrogatoire consiste en une forme de procès où tous les proches du défunt auront à se défendre de leur participation ou de leur responsabilité dans la mort de leur parent. Il permet également de déceler des alliances contractées par des puissances invisibles et restées méconnues des proches. À propos de ce rituel en pays Joola Felup, Odile Journet et André Julliard précisent que « l'interrogatoire consiste certes à expliciter officiellement - à proclamer publiquement en quelque sorte - les causes du décès que chacun connaît par avance. Mais il sert surtout à désamorcer les risques d'un éventuel cycle de violences (et pas seulement à travers la sorcellerie) qui pourrait s'enclencher après la mort (...). L'interrogatoire procure encore une sorte d'apaisement : il ramène subtilement, par le raisonnement moral, la cause du décès aussi horrible soitelle à la volonté de Dieu (ce qui n'exclut pas les obligations de sacrifices réparateurs pour la famille)» $(1989$, p. 153).

L'absence de ces acteurs clés dans le contexte d'immigration entraîne une délocalisation de certaines séquences. Par exemple, dans le cadre des funérailles manjak en migration, l'interrogation du mort ne peut être réalisée en France pour des raisons liées à l'espace dans lequel les rituels sont réalisés mais aussi parce qu'il nécessite un savoir-faire dont ne disposent pas les immigrés. En effet, les porteurs, investis d'une mission délicate, doivent répondre à des qualités très précises. Ainsi, elle sera réalisée au village lors du retour de la valise et un vêtement viendra suppléer l'absence du corps du défunt, le plus souvent inhumé en France.

Dans d'autres cas, on assiste à une redistribution des rôles. En migration, l'habilleur des morts est souvent remplacé par un membre de la communauté désigné, non pas par rapport à son statut social, mais au regard de son savoir-faire. Il est souvent choisi parmi les aînés ou ceux qui ont migré plus tardivement, parce qu'ils connaissent les gestes à accomplir, les paroles à prononcer pour purifier le corps, lui rendre sa souplesse et pour apaiser le défunt. De plus, au cours des funérailles, les responsabilités financières sont désormais confiées à une personne de confiance avec qui les proches peuvent s'exprimer librement et ouvertement, généralement l'aîné des enfants ou un ami intime du (de la) défunt(e).

24 La délocalisation de certaines séquences induit une déstructuration de l'enchaînement séquentiel des rites funéraires. De plus, la situation d'immigration oblige à redéfinir les fonctions au cas par cas, en fonction de l'éventail des relations disponibles et de la représentation des différentes fractions sociales qui ont un rôle à tenir, mais aussi des relations valorisées et du système de valeurs privilégié par les proches du défunt présents sur le lieu du décès. L'observation des mécanismes sociaux à l'œuvre au cours des cérémonies funéraires permet de saisir des changements dans les valeurs de référence et atteste de la volonté des acteurs d'une part, de rompre avec certaines formes de contraintes sociales et d'obligations et d'autre part, de compenser la perte de compétence rituelle par le recours à de nouveaux acteurs qui disposent des compétences propres au contexte migratoire.

Le village prestataire de rituels

25 La gestion rituelle transnationale, la perte de compétence des acteurs rituels en migration et les difficultés liées au cadre de réalisation confèrent au village d'origine un rôle clé dans l'accomplissement des devoirs funéraires. Au village, la famille dispose de plus de temps, d'une meilleure maîtrise des dispositifs rituels et l'aménagement spatial est plus 
propice à la célébration des veillées, des funérailles et des rites de commémoration mais aussi à la reproduction du système d'obligations réciproques. L'interrogatoire des morts, les grandes funérailles et les rites d'intronisation des ancêtres ont lieu dans le village d'origine. L'autel des ancêtres n'a pas été transféré dans le nouveau pays de résidence et reste lié à la maison familiale dans le pays d'origine. C'est d'ailleurs un vecteur de liens très puissant entre les émigrés et le village d'origine. De leur côté, les immigrés ont mis en place des «caisses d'enterrement " et ont organisé un système de participation et d'obligations réciproques codifié entre villages et familles dont la fonction est d'assurer une bonne organisation des cérémonies funèbres en France mais également dans le pays d'origine en offrant un soutien matériel et surtout financier de taille à la famille endeuillée. Actuellement à Marseille, les caisses d'enterrement existent sous la forme du regroupement de plusieurs villages appartenant au même poste administratif du pays manjak. Ces caisses sont des structures informelles (c'est-à-dire qu'elles ne sont pas déclarées en Préfecture) qui entretiennent entre elles des liens de solidarité et ont des obligations réciproques. À l'occasion d'un décès, les différentes caisses de funérailles siègent en une assemblée extraordinaire au cours de laquelle chacune donne la participation préalablement fixée. Une délégation sera chargée de l'apporter à la famille endeuillée dans les plus brefs délais.

Dans le contexte d'immigration, la famille restée dans le pays d'origine devient prestataire de rituels en échange des sommes versées par la communauté immigrée et par l'entourage et ramenées par l'accompagnateur de la valise mortuaire. Les migrants mettent en avant leur fonction de financeurs des rituels qui ont lieu au village tandis que l'exécution est confiée à un membre de la famille qui joue les intermédiaires. Les membres de la famille restés au village ou ceux qui y effectuent des séjours réguliers et de longue durée deviennent les dépositaires d'un savoir-faire rituel menacé par la migration et sont investis d'une mission visant a priori à la pérennisation des croyances, des systèmes symboliques et sociaux et des pratiques coutumières. Le village devient alors « la base arrière de la tradition où, par procuration, la reproduction symbolico-religieuse continue d'être assurée » (Hassoun, 1993, p. 199). En investissant le village pour réaliser une partie des rituels funéraires par l'intermédiaire d'un ou plusieurs représentants de la « communauté » migrante, les immigrés témoignent de leur volonté de préserver leurs «traditions » et par conséquent de la priorité symbolique ultime accordée au pays d'origine.

Si les immigrés ont l'impression, à travers ces échanges cérémoniels, de contribuer à la pérennisation d'un système symbolico-religieux, ils participent également, sans toujours en avoir conscience, à son bouleversement et aux mutations des pratiques cérémonielles dans les régions d'origine car cette relation n'est pas sans conséquences. En effet, la gestion rituelle dans les villages n'est pas épargnée par certains changements qui affectent les cérémonies familiales en milieu urbain et dans les zones de forte émigration, liés à la surenchère et l'ostentation. Or, les immigrés condamnent ces évolutions et sont généralement choqués par le sens de ces changements. Mais paradoxalement, ils entretiennent voire même aggravent cette situation qu'ils ne maîtrisent pas, ou en tous cas ne cautionnent pas, à travers les sommes considérables injectées pour financer les funérailles et rembourser les dettes contractées par la famille qui accompagnent le retour de la valise mortuaire.

Les dispositifs rituels en migration montrent que le village reste au centre des relations avec l'au-delà, les ancêtres et de celles entre les vivants et les morts. Ils donnent la preuve 
d'un enracinement qui, à défaut d'être concret, est symbolique et émotionnel. Le lieu d'origine est (ré)investi symboliquement et les migrants, en dépit de leur situation qui va parfois plus dans le sens d'un ancrage et d'une « intégration » en France, attestent par-là que le village reste une référence centrale. Toutefois, l'analyse du dispositif funéraire migrant montre bien que cet investissement symbolique est finalement double: l'inhumation en France participe à une forme d'ancrage sur le territoire d'immigration tandis que le retour symbolique de la valise mortuaire, la mise en œuvre du processus d'ancestralisation au village attestent de liens spirituels très forts avec le village d'origine, principalement par le biais des ancêtres.

Conclusion

29 Les rituels funéraires manjak en migration se déroulent sur une scène plurielle. Cette disjonction, à laquelle s'ajoutent les contraintes pratiques, juridiques, sociales liées au contexte d'immigration, empêche une reproduction à l'identique que ce soit en France ou dans le village d'origine. Ils subissent d'inévitables altérations et transformations. Les rituels funéraires occasionnés par le décès d'un migrant ne sont ni une reproduction fidèle de ceux qui ont ou avaient cours dans les régions d'origine, étant donné le contexte social et le cadre spatial dans lesquels ils se réalisent, ni une réplique des rituels funéraires pratiqués en France en milieu urbain, du fait du maintien de certaines spécificités. Ils prennent des formes inédites qui ne sont de l'ordre ni de la résistance, ni de l'acculturation mais plutôt d'une logique de dédoublement qui autorise «la cohabitation des contraires» (Mary, 2000) et donne à ces dispositifs une continuité, renforcée par l'économie du symbolique et de la dette. Les rituels funéraires manjak en migration se jouent sur un registre particulier : celui de l'entre-deux culturel, spatial et temporel, caractéristique de la situation migratoire.

30 La situation migratoire confère également aux rituels une nouvelle symbolique: les funérailles ne sont plus uniquement destinées à l'accompagnement du défunt dans son devenir post-mortem et à la restauration symbolique de la communauté des vivants, elles marquent également le retour définitif d'une migration d'abord pensée comme provisoire et axée sur le projet de retour au village et symbolisent de fait l'entre-deux qui la caractérise. Les funérailles célèbrent en quelque sorte la cohésion d'un groupe, au-delà des distances qui les séparent et s'inscrivent dans un espace qui dépasse largement les frontières d'ici et de là-bas. Finalement, les rituels funéraires témoignent de la volonté des acteurs de préserver une forme de continuité lignagère, menacée par l'immigration. Ils attestent également d'un renversement des rapports de dépendance. En effet, alors que dans la vie quotidienne l'économie villageoise et familiale est tributaire des envois et de la participation des immigrés qui assument en grande partie les dépenses, notamment les dépenses cérémonielles, et ont, en extrapolant un peu, un " pouvoir de (sur)vie et de mort » sur leurs dépendants, la gestion rituelle des funérailles renverse cette allégeance et ce sont les immigrés qui deviennent tributaires de leurs parents restés au village qui garantissent le bon déroulement des rituels et assurent de ce fait le devenir post-mortem de ceux qui sont morts au loin. 


\section{BIBLIOGRAPHIE}

Brisebarre Anne-Marie, dir., La fête du mouton. Un sacrifice musulman dans l'espace urbain, Paris, Éditions du CNRS, 1998.

DEssing Nathal, Rituals of Birth, Circumcision, Marriage and Death among Muslims in the Netherlands, Louvain, Peeters, 2001.

Diop Amadou Moustapha, «Tradition et adaptation dans un réseau de migration sénégalais : la communauté Manjak en France », thèse d'anthropologie, Paris, EHESS, 1981.

Diop Amadou Moustapha, Société manjak et migration, Besançon, Demontrond, 1996.

Hérault Laurence, « L'espace de la variation. Étude des variations et des transformations de deux rites de passage dans le Haut-Bocage vendéen », thèse de nouveau régime en ethnologie, Université de Provence, 1992.

Hassoun Jean-Pierre, « La migration des Hmong en Occident. Rituels funéraires, "prix de la fiancée : stratégies d'adaptation des premières années” ", Ethnologie Française, XXIII (2), 1993.

Hassoun Jean-Pierre, Hmong du Laos en France : changement social, initiatives et adaptations, Paris, PUF, 1997.

Journet Odile, Julliard André, « Interrogatoire du mort en pays Joola Felup », Systèmes de pensée en Afrique noire, 9, 1989, p. 135-153.

Mary André, Le bricolage africain des héros chrétiens, Paris, Cerf, 2000.

Petit Agathe, «La mort au loin. Les pratiques funéraires des migrants africains en France », thèse de doctorat en anthropologie sociale et ethnologie (sous la direction d'André Mary), EHESS

Marseille, 2002.

Petit Agathe, «Le rapatriement post-mortem ou l'ultime retour des "gens du Fleuve" »; Hommes et migration, 1236, mars-avril 2002, p. 44-52.

Ségalen Martine, Rites et rituels contemporains, Paris, Nathan, 1998 (collection 128).

Sibony Daniel, Entre-deux. L'origine en partage, Paris, Seuil, 1991.

Teixeira Maria, « Changement social et contre-sorcellerie féminine chez les Manjak de Canchungo émigrés Ziguinchor : les réponses du Bëpene et du Kasara », thèse de doctorat en anthropologie, EHESS, 1996.

Teixeira Maria, Rituels divinatoires et thérapeutiques chez les Manjak de Guinée-Bissau et du Sénégal, Paris, L'Harmattan, 2001.

\section{NOTES}

1. Il existe toutefois deux travaux de références, ceux d'A.M. Diop (1996) et M. Teixeira (1996 et 2001).

2. La « communauté chrétienne africaine » de Marseille est actuellement dirigée par deux prêtres ouvriers et un sacristain manjak. Ils organisent une messe mensuelle, un 
pèlerinage annuel, des sorties, une chorale... La communauté chrétienne fédère environ 800 familles dont une majorité de Manjak et fonctionne comme un lieu de centralité, d'échanges et de célébration des cérémonies familiales. Cependant, les responsables tentent depuis quelques années de décentraliser la communauté en dirigeant les croyants vers les paroisses de leurs lieux de résidence, notamment pour l'organisation des cérémonies familiales (messes de baptême, mariage, enterrement, anniversaire de décès...).

3. Cet espace propose des concessions individuelles de 15 et 30 ans. En fait, cette dénomination euphémisée couvre une réalité autre et cet espace ressemble davantage à un « immeuble cimetière », ce qui lui vaut l'appellation d'« HLM de la mort ». Il s'agit d'un bâtiment qui ressemble dans son aspect extérieur à un immeuble volumineux de quatre étages. À chaque niveau, les murs sont couverts d'au moins six rangs de niches individuelles qui peuvent recevoir un cercueil. Il n'y a ici ni pierre tombale, ni stèle, seulement une plaque commémorative à laquelle sont parfois accrochées quelques fleurs artificielles. De nombreux ouvrages et articles de presse font allusion à ce surprenant type de sépulture en élévation ou à étages, construit à Marseille, qualifié de « mur de la mort ", qui représente un nouveau type de conception du cimetière adapté à la pénurie d'espace.

4. Notons que le calendrier des messes est variable et que cette première messe du souvenir peut, dans certains cas, être célébrée un mois après le décès, voire six mois selon les moyens financiers de la famille car chaque messe est suivie d'une réception. 5. Voir à ce propos l'article d'Agathe Petit « Le rapatriement post-mortem ou l'ultime retour des "gens du Fleuve" ", Hommes et migration, 1236, mars-avril 2002, p. 44-52.

\section{RÉSUMÉS}

Cette contribution s'intéresse à l'aménagement des rituels funéraires des migrants manjak en France. Les pratiques funéraires qui ont cours dans la culture d'origine, elles-mêmes susceptibles de se modifier, ne peuvent être transposées à l'identique du fait des nombreuses contraintes liées au nouveau cadre de vie qui combine urbanisation et migration. Ces pratiques confrontent ou font coexister deux systèmes de références culturelles différents, se jouent en plusieurs temps, sur plusieurs espaces (ici et là-bas, entre ici et là-bas). Il s'agit d'une situation originale qui bouleverse les normes et compromet leur unité, entraîne une déstructuration et une discontinuité dans le déroulement séquentiel des funérailles et oblige à des négociations et à des remaniements.

The article deals with the adaptation of the funerary rituals of the Manjak migrants in France. The funerary traditions of the original culture, which are themselves likely to undergo adaptations, cannot be transposed as such given the many constraints linked to the new environment created by immigration and urbanization. These traditions confront or bring together two different systems of cultural references, and they are performed at different times, in different places (here and over there, between here and there). This is an original situation which upsets existing norms and jeopardizes their unity; it leads to a dislocation and discontinuity of the ordered development of the funerals and forces negotiations and revisions. 
Esta contribución se aboca a la construcción de los rituales funerarios de los migrantes manjak en Francia. Las prácticas funerarias que tuvieron lugar en la cultura de origen, éstas mismas susceptibles de ser modificadas, no pueden ser transpuestas de manera idéntica, debido a las numerosas determinaciones del nuevo contexto que combina urbanización y migración. Estas prácticas confrontan o hacen coexistir dos sistemas de referencias culturales diferentes, se juegan en varios tiempos, en varios espacios (aquí y allá, entre aquí y allá). Se trata de una situación original que transforma las normas y compromete su unidad, acarrea una desestructuración y una discontinuidad en el desarrollo secuencial de los funerales y obliga a negociaciones y a reacomodamientos.

INDEX

Mots-clés : accommodation, Afrique de l'Ouest, bricolage, migration, pratiques funéraires

\section{AUTEUR}

AGATHE PETIT

IRTS Paca-Corse 